\title{
Gestión del talento en la empresa española. Rol del departamento de recursos humanos
}

\author{
Gallardo-Gallardo, Eva* \\ González-Cruz, Tomás** \\ Martínez-Fuentes, Clara*** \\ Pardo-del-Val, Manuela****
}

\begin{abstract}
Resumen
El propósito de este artículo es doble: explicar, con base en la revisión de la literatura especializada, el concepto de talento y el rol del departamento de recursos humanos en la gestión de talento; y analizar, mediante el método Delphi, y para las empresas españolas, la percepción de los expertos consultados sobre la definición de talento, así como también, el rol del departamento de recursos humanos y el efecto de la actual crisis en la gestión del talento. Los resultados evidencian que: talento es entendido como una mezcla de competencias y compromiso que las personas demuestran mediante un desempeño excepcional, coincidiendo con la conceptuación más aceptada recientemente; el departamento de recursos humanos sigue sin estar vinculado al diseño e implementación de la estrategia de la empresa y, que en tiempos de crisis toda acción emprendida por dicho departamento queda supeditada a los costes, en línea con lo apuntado por la literatura.
\end{abstract}

Palabras clave: Definición de talento; gestión del talento; método Delphi; empresas españolas

Recibido: 20-07-11. Aceptado: 30-03-12

* Licenciada en administración y dirección de empresas (Universidad de Barcelona). Profesora asociada del departamento de economía y organización de empresas. Facultad de Economía y Empresa. Universidad de Barcelona. España. e-mail: eva.gallardo@ub.edu. Autor para la correspondencia.

** Doctor en ciencias económicas (Universidad de Valencia). Profesor titular del departamento de dirección de empresas "Juan José Renau Piqueras". Facultad de Economía. Universidad de Valencia. España. e-mail: tomas.gonzalez@uv.es.

*** Doctora en ciencias económicas (Universidad de Valencia). Profesora Titular del departamento de dirección de empresas "Juan José Renau Piqueras". Facultad de Economía. Universidad de Valencia. España. e-mail: clara.martinez@uv.es.

**** Doctora en ciencias económicas. Universidad de Valencia. Profesor titular del departamento de dirección de empresas “Juan José Renau Piqueras”. Facultad de Economía. Universidad de Valencia. España. e-mail: manoli.pardo@uv.es. 


\title{
Talent Management in Spanish Firms: Role of the Human Resource Department
}

\begin{abstract}
The aim of this paper is twofold: first, to explain, the concept of talent and the role of the human resources department in talent management, based on a literature review; and second, to analyze, using a Delphi method, expert perception about the definition of talent as well as the role of the human resources department and the effect of the current crisis on talent management in Spanish firms. Results show that talent is understood as a mixture of competences and commitment that people demonstrate through exceptional performance, coinciding with the most recently accepted conceptualization; the human resources department is still not linked to the design and implementation of the firm's strategy; and, in times of crisis, any action undertaken by this department depends on costs, in line with what is indicated in the literature.
\end{abstract}

Keywords: Definition of talent, talent management; Delphi Method, Spanish firms.

\section{Introducción}

No hace mucho tiempo, Lewis y Heckman (2006) afirmaban que una revisión no muy profunda de la literatura sobre gestión del talento llevaría a concluir que se trataba de un tema de investigación de moda y en auge. Desde entonces, el interés sobre este tema ha crecido exponencialmente, quizás porque la gestión del talento (GT) se considera clave para el éxito de la organización actual al poder generar una ventaja competitiva mediante la identificación, desarrollo y recolocación de los empleados con talento (lles et al., 2010). No obstante, pese a la creciente popularidad de este tema y como en otras áreas de reciente desarrollo, la definición y concreción en pautas de acción de la GT es todavía difusa (Collings y Mellahi, 2009; González et al., 2009; Lewis y Heckman, 2006).

En 2006, un artículo publicado en The Economist afirmaba que las empresas no sabían cómo definir talento, cuanto menos gestionarlo (The Economist,
2006). Un año después, las diferentes opiniones existentes sobre talento llevaron al Chartered Institute of Personnel and Development (CIPD) -el mayor organismo europeo de recursos humanos (RR.HH.) y desarrollo profesional- a sugerir que "el punto de partida de cualquier investigación sobre gestión del talento debía ser inevitablemente una exploración sobre lo que significa talento" (2007: 3). De acuerdo a Maxwell y MacLean (2008), al centrarse la GT en el 'talento', este concepto debe ser definido.

De la revisión de la literatura especializada emergen un sinfín de definiciones y opiniones sobre el término talento. Luego, se puede afirmar que actualmente no existe consenso sobre qué se entiende por talento. De hecho, en la literatura se presentan prácticas tradicionales de RR.HH. como medio para gestionar el talento en las organizaciones sin aclarar previamente si la unidad de análisis son las personas y/o sus características individuales. Luego, se hace necesaria una reflexión sobre la naturaleza y definición 
Gestión del talento en la empresa española. Rol del departamento de RR.HH.

Gallardo-Gallardo, E.; González-Cruz, T.; Martínez-Fuentes, C. y Pardo-del-Val, M.

del concepto talento en el ámbito empresarial con el fin, no sólo de poderlo gestionar de forma eficiente y eficaz, sino también para poder diferenciar la GT de la dirección de RR.HH.

A nivel organizativo, la coordinación e implementación de las prácticas de RR.HH. está a cargo del departamento de RR.HH. Departamento que en el pasado reciente ha sido relegado a un rol más bien burocrático y administrativo, pero al que ahora se le pretende asignar un papel de carácter estratégico dada la importancia creciente de las personas como fuente de ventaja competitiva. Son varios los autores (Lawler, 2008; Ulrich y SmaIlwood, 2005) que abogan por un departamento y unos profesionales de RR.HH. que vinculen su trabajo con el valor para los accionistas, es decir, que estén imbricados en el núcleo de la estrategia de la empresa y sean un facilitador de la misma.

Además, al departamento de $\mathrm{RR}$.HH. se le presupone un rol de soporte y guía en el diseño e implementación de la GT de modo que cumpla con las necesidades de la organización (Tansley et al., 2007). Cabe decir que las evidencias que se tienen hasta el momento acerca del rol del departamento de RR.HH. en la GT derivan principalmente del estudio de prácticas aplicadas en grandes empresas estadounidenses e inglesas. Principalmente, entidades financieras (Bank of America, Standard Chartered PLC), consultoras (PricewaterhouseCoopers LLP, Tenon), empresas de comunicaciones (3 Monkeys), empresas de software (Autodesk), punto com (Google) y empresas de hostelería y restauración (Gordon Ramsay Holdings) -véanse, por ejemplo,
Effron y Ort (2010); Tansley et al. (2007) y Thorne y Pellant (2007).

En España la GT es un campo de estudio novedoso tanto para académicos -el único trabajo publicado del que tenemos constancia es González et al. (2009)-como para directivos. Además, el informe Top-talento: Estudio de la gestión del talento en la empresa española pone de manifiesto que sólo en un $4,5 \%$ de las empresas españolas existe mentalidad o 'mirada' hacia el talento (Luna, 2010). Lo cual es preocupante en los tiempos de crisis actuales, cuando los gurús empresariales aconsejan recurrir a la creatividad y al talento de los miembros de la organización para afrontar la coyuntura con éxito y garantizar la supervivencia de la empresa.

Así, el propósito de este artículo es doble: explicar el concepto de talento y, el rol del departamento de RR.HH. en la GT mediante una revisión exhaustiva de la literatura; y, analizar, utilizando el método Delphi, el concepto de talento, el rol del departamento de recursos humanos y el impacto de la crisis en la GT en el caso de las empresas españolas.

Dada la naturaleza exploratoria de los objetivos de investigación y la escasez de estudios en el ámbito español, se ha considerado el método Delphi como el instrumento idóneo para la obtención de datos. Se trata de una técnica que tiene como objetivo la obtención de un consenso lo más fiable posible en la opinión de un grupo de expertos (denominado, panel) a través de cuestionarios estructurados y gracias a una retroalimentación controlada (Dalkey y Helmer, 1963). Para ello el investigador se centra en tres elementos (Dalkey et al., 1972): el anonima- 
to en la respuesta, la iteración y retroalimentación controlada que anima a converger en las opiniones y, la respuesta del grupo en forma estadística (a los expertos se les presenta no sólo el punto de vista de la mayoría sino todas las opiniones indicando el grado de acuerdo que se ha obtenido).

Obviamente, la calidad de los resultados obtenidos mediante este método de análisis depende, en gran medida, de la selección de los expertos. Por ello, en esta investigación, la elección del panel de expertos se ha realizado en base a sus amplios conocimientos y demostrada experiencia profesional en consultoría y dirección de RR.HH. en el ámbito español. Siguiendo estos criterios, en abril de 2009 , se seleccionaron ocho expertos ${ }^{1}$ a los que se les explicó: el propósito del estudio, en qué consistía el método Delphi (con el fin de obtener previsiones fiables), así como también, el código ético relativo al tratamiento y posterior difusión de la información recogida en el estudio. De este modo se consiguió su compromiso de colaboración.

Los investigadores actuaron como moderadores y elaboraron un cuestionario con tres grandes bloques de preguntas: El primer bloque estaba conformado por tres preguntas abiertas y tres de respuesta numérica (cada una de ellas desglosada en diferentes ítems, siguiendo una escala Likert de 5 puntos) referidas al concepto de talento. Un segundo bloque, trataba las prácticas de RR.HH. y el rol del departamento de RR.HH. en las empresas españolas. Estaba compuesto por una pregunta abierta, cuatro de respuesta numérica (desglosadas en diferentes ítems cada uno de ellos siguiendo una escala Likert de 5 puntos) y una en la que se pedía una ponderación (repartir 100 puntos) entre diferentes funciones que pueden desempeñar los departamentos de RR.HH.

El último bloque de preguntas versaba sobre la GT en las actuales circunstancias de crisis. Constaba de trece ítems que valoraban, según una escala Likert de 5 puntos y para tres períodos de tiempos distintos (pre-crisis, crisis y post-crisis), las estrategias y prácticas de reclutamiento y selección utilizados por las empresas españolas. También, en este bloque había dos preguntas de carácter prospectivo. Una sobre la posible influencia de la actual situación económica en una serie de roles del departamento de RR.HH. y, la otra, sobre cómo debería cambiar el enfoque de las empresas respecto a la dirección de RR.HH. para afrontar la actual crisis. Estas dos últimas series de preguntas se valoraban siguiendo una escala de -3 (máxima reducción) a +3 (máximo aumento).

Se realizó una primera circulación del cuestionario, vía e-mail, cuyo objetivo fue recoger las opiniones iniciales de los

1 Dalkey et al. (1970), sugieren un mínimo de siete expertos ya que el error disminuye significativamente con la adición de un experto hasta siete, de ahí en adelante, la reducción del error no está compensada por los costes adicionales. Asimismo, recomiendan cómo máximo un panel de 30 expertos. 
Gestión del talento en la empresa española. Rol del departamento de RR.HH.

Gallardo-Gallardo, E.; González-Cruz, T.; Martínez-Fuentes, C. y Pardo-del-Val, M.

expertos. Tras ella, se calculó la mediana y el recorrido intercuartílico para cada una de las respuestas numéricas y se acumularon las respuestas de las preguntas abiertas. Con un periodo de carencia de diez días, se hizo una segunda circulación del cuestionario, en la que a cada experto se le envió, junto a sus respuestas previas, el cálculo de la mediana y del recorrido intercuartílico para cada una de las preguntas, así como, la lista de respuestas para las preguntas abiertas. De este modo, cada experto podía contrastar su opinión con la del resto del grupo. El proceso finalizó en esta segunda circulación ya que se alcanzó una dispersión aceptable en las respuestas.

\section{Análisis de la definición de talento en la literatura empresarial}

A partir de la revisión de la literatura sobre GT se pueden agrupar en tres categorías las definiciones del término talento: aquellas que lo entienden como las personas de una organización; las que consideran que hace referencia únicamente a un grupo de personas con alto rendimiento o potencial dentro de la organización; y, por último, aquellas definiciones que apelan al concepto de talento como las habilidades, capacidades y/o competencias de una persona.

\subsection{Talento entendido como las personas de la organización}

Lewis y Heckman (2006: 141) en su revisión crítica sobre la literatura de gestión del talento afirman que "talento es fundamentalmente un eufemismo de per- sonas". De hecho, son muchos los autores (véanse por ejemplo: Cheese et al., 2008; Lawler, 2008; O'Reilly y Pfeffer, 2000) que utilizan los términos talento y personas (o recursos humanos) de la organización como sinónimos. Dicha concepción llevaría a una interpretación de la GT similar a la dirección de RR.HH. que, según lles et al. (2010), implicaría una colección de típicas prácticas de RR.HH. como el reclutamiento, la selección, formación y sistemas de recompensa. Luego, reemplazar la palabra "personas" por "talento" puede servir para renombrar las prácticas de RR.HH. y hacerlas parecer nuevas y frescas, pero no ayuda a mejorar el conocimiento sobre la gestión estratégica y efectiva del talento (Lewis y Heckman, 2006).

\subsection{Talento entendido como un grupo de personas con un alto rendimiento y/o potencial}

Stahl et al. (2007) afinan la definición de talento al referirse como tal al grupo de empleados que puntúan alto en los rankings en términos de capacidad y desempeño. Normalmente, a dichos empleados se les llaman "A players" o de alto rendimiento (high-performers), conforman el talent pool y se les suele considerar el futuro de la organización. Ligando de forma más explícita talento con desempeño organizativo, Tansley et al. (2007: 8) proponen la siguiente definición de talento: "aquellos individuos que pueden marcar una diferencia en el desempeño organizativo bien mediante su inmediata contribución como en el largo plazo, demostrando sus altos niveles de potencial". 
En cualquier caso, se trata de una interpretación basada en la segmentación y diferenciación de la fuerza de trabajo. Fundamentalmente, se basa en identificar a las personas con un alto potencial y/o resultados extraordinarios. Si se trata de personas de fuera de la organización el reto se centra en atraer, reclutar, retener y desarrollar a esas estrellas -lema de la famosa guerra por el talento anunciada a finales de los 90 por los consultores de McKinsey. Si son personas de dentro de la organización, el reto principalmente residirá en retenerlas y desarrollarlas. Luego, la GT consistirá en utilizar las mismas herramientas de selección, reclutamiento, retención y formación que la dirección de RR.HH. pero, en este caso, focalizadas en un segmento específico de la fuerza de trabajo, en los talentosos -aquellos con un alto rendimiento y/o un futuro potencial.

No obstante, esta interpretación exclusivista con un invariable énfasis en el alto desempeño del individuo no está exenta de críticas. Por ejemplo, su carácter selectivo puede causar desmotivación y sentimiento de exclusión entre aquellos que no pertenecen a ese talent pool. Además, según Pfeffer (2001) un énfasis excesivo en el desempeño individual disminuye el trabajo en equipo y crea una competitividad interna destructiva que retarda el aprendizaje y la diseminación de mejores prácticas en la compañía. Finalmente, en la medida en la que se utilizan las consecuencias -los resultados extraordinarios- como indicadores para identificar a los individuos con talento, se puede llegar a enfoques de carácter tautológico.

\subsection{Talento entendido como habilidades, capacidades y/o competencias}

La anterior interpretación de talento hace referencia a un grupo de personas con unas habilidades excepcionales, un alto desempeño y/o potencial. En otras palabras, está definiendo a las personas con talento. Ergo, talento, en su concepción más concreta, hace referencia a esas habilidades excepcionales que una persona posee. Por ejemplo, Michaels et al. (2001: xiii), quizás en una de las definiciones más citadas, se refieren al talento directivo como "una combinación de una aguda mentalidad estratégica, habilidad directiva, madurez emocional, habilidades de comunicación, habilidades para atraer e inspirar a otra gente con talento, instintos emprendedores, habilidades funcionales y la habilidad de ofrecer unos resultados".

Goffee y Jones (2007: 72) se refieren al talento como "aquellas ideas, conocimientos y habilidades que proporcionan a la persona el potencial para producir un valor desproporcionado con los recursos que sus organizaciones le facilitan". Mientras que, Silzer y Dowell (2010: 14) proponen una segunda definición de talento que hace referencia a las habilidades individuales (tanto innatas como adquiridas) y a lo que la persona es capaz de hacer o contribuir en la organización. De la creencia de la organización y, sobre todo entre la alta dirección, sobre si el talento es innato o no, se derivarán las acciones pertinentes: apostar por la selección y reclutamiento (comprar el talento) o bien, por el desarrollo y formación (hacer gente con talento). 
Gestión del talento en la empresa española. Rol del departamento de RR.HH.

Gallardo-Gallardo, E.; González-Cruz, T.; Martínez-Fuentes, C. y Pardo-del-Val, M.

Si bien la mayoría de las definiciones de talento establecen algún vínculo entre habilidades y desempeño, al hablar de talento cobran cada vez más importancia las competencias y las actitudes -en concreto, el compromiso- de las personas. Por ejemplo, Dave Ulrich (2007) define talento como "competencia x compromiso x contribución"2 . De este modo, no sólo pone el énfasis en las habilidades y conocimientos de las personas, sino también en sus actitudes y en su alineación con la organización. Luego, su gestión deja de ser algo indefinido, para pasar a ser tangible y estratégica.

En 2008, Juan Carlos Cubeiro, socio-director de IDEO Business y conferenciante de prestigio internacional, definió talento como capacidad por compromiso en un determinado entorno. Entendiendo por capacidad, aptitud (conocimientos y habilidades de la persona) más actitud (estado de ánimo mostrado en los comportamientos). Pese a que no profundiza en el resto de los componentes de la ecuación del talento, conviene subrayar el hecho de que enfatiza el contexto organizativo a la hora de hablar de talento.

En la misma línea, González-Cruz et al. (2009: 22) definen talento como "aquel conjunto de competencias que, desarrolladas y aplicadas, permiten a un sujeto desempeñar un determinado papel de forma sobresaliente". Al igual que Cubeiro, estos profesores también resaltan la importancia de contextualizar la definición de talento, en este caso, acotándola al rol que la persona esté ejecutando.

Dentro de esta interpretación de talento se observan dos posturas bien diferenciadas, si bien en ambas existe un claro vínculo entre talento y resultados extraordinarios. Por un lado, la de aquellos autores que abogan por un talento circunscrito a las habilidades excepcionales de las personas (tanto las consideradas innatas, y por tanto de difícil gestión, como las adquiridas o adquiribles mediante planes formativos). Por otro lado, la de aquellos autores que sugieren un concepto de talento basado no sólo en conocimientos y habilidades (por muy excepcionales que sean) sino también en actitudes (principalmente, el compromiso). Convirtiendo de este modo al concepto de talento en algo más tangible y gestionable mediante las técnicas y herramientas propuestas por la literatura de gestión por competencias. los puestos de trabajo tanto de hoy como futuros, que hacen que la gente tome decisiones acertadas y entregue resultados en la manera correcta. Luego, indirectamente está haciendo referencia al rendimiento de las personas. Por otro lado, este autor considera el compromiso como "la voluntad de los empleados para dar su energía discrecional en beneficio de la organización" (2007: introducción). De esta forma está enfatizando el papel de la organización al hablar de talento, pues la organización se beneficiaría de él. Por último, considera que la contribución, "aparece cuando los empleados sienten que sus necesidades personales se satisfacen mediante su participación en la organización" (Ulrich 2007: introducción). Para este autor, los tres términos de la ecuación son multiplicativos de tal forma que si uno de ellos no existe, el talento es nulo. 
En cualquier caso, una exhaustiva revisión de la literatura muestra la diversidad y heterogeneidad de las aportaciones que tratan de definir el término talento. Por ello parece pertinente pulsar la opinión de los expertos a la hora de establecer una definición operativa.

\section{Rol y prácticas del departamento de RR.HH. en la gestión del talento: algunas consideraciones en tiempos de crisis}

Tal y como se ha mencionado anteriormente, en la revisión de la literatura es frecuente encontrar referencias a prácticas de RR.HH. al hablar de la GT. Así, en función del conjunto de prácticas adoptadas y de la conexión de éstas con el proceso de formulación e implementación de la estrategia se puede extrapolar el rol del departamento de RR.HH. Diversos casos de estudio apuntan a la necesidad de que el departamento de RR.HH. desempeñe un papel de guía y apoyo a los directivos y mandos intermedios con el fin de lograr una buena implantación del sistema de GT (véase, Tansley et al. 2007).

En paralelo, otros autores (por ejemplo: Lawler, 2008; Ulrich y SmaIlwood, 2005) reclaman para el departamento de RR.HH. un rol más estratégico -vinculado con los procesos de formulación e implementación de la estrategiaen contraposición con el rol meramente administrativo que durante años ha tenido, pues, como afirma Gubman (1998) la fuerza de trabajo es la única cosa suficiente y necesaria para ejecutar la estrategia. Es más, Lawler (2008) y Ulrich y
Brockbank (2005) apuestan por un departamento de RR.HH. 'socio estratégico del negocio'. No obstante, la realidad sigue siendo muy diferente en la mayoría de las empresas. Farley (2005) describe la difícil situación en la que se encuentra el departamento de RR.HH. en muchas organizaciones teniendo muchas cosas que hacer pero sin poder o credibilidad para poder hacerlas.

De acuerdo con Blass (2009), las prácticas de GT más efectivas serán aquellas específicas de cada organización al responder a un contexto organizativo y un capital humano concreto. No obstante, es posible identificar un conjunto de prácticas y procesos generales que determinan el rol estratégico del departamento de RR.HH. al alinear las necesidades de la organización con los esfuerzos y desempeño de las personas. Entre ellas destacan: la selección y reclutamiento basado en un modelo de competencias, el performance management, la retroalimentación $360^{\circ}$, planificación de la sucesión, cuestionarios para conocer el nivel de motivación y compromiso.

Este tipo de prácticas y procesos que pretenden alinear necesidades de la organización con el desempeño de los individuos toman, si cabe, más relevancia en los tiempos de crisis dónde los recursos son escasos, y las empresas para sobrevivir deben saber detectar, reconocer y explotar las oportunidades que se les presenten. Precisamente, en una época de crisis como la que se está viviendo, es cuando la gestión del talento se vuelve más importante. De hecho, "la importancia de la gestión del talento en la actual situación de crisis reside en facilitar a las 
Gestión del talento en la empresa española. Rol del departamento de RR.HH.

Gallardo-Gallardo, E.; González-Cruz, T.; Martínez-Fuentes, C. y Pardo-del-Val, M.

empresas la consecución de sus necesidades clave tanto a corto como a mediolargo plazo" (CIPD, 2009b: 9).

Un estudio del CIPD (2009a), elaborado al inicio de la recesión económica, subraya cómo la GT de algunas empresas, sobre todo del sector privado, se ha visto afectada debido a recortes presupuestarios. Dichos recortes han afectado principalmente al presupuesto de formación y desarrollo de la organización y, a la compensación (con restricciones y/o congelaciones en las subidas de sueldo y/o bonos y, con la implantación de un sistema de pago por objetivos). Las empresas han abogado por: congelar las contrataciones; focalizar su atención en la retención de las personas con alto desempeño (asegurando que su compensación no se haya visto perjudicada, sino al contrario); y, por el desarrollo de talento dentro de la propia empresa, apostado sólo por programas de desarrollo imprescindibles (por ejemplo, los dirigidos a directivos para ayudarles a liderar, motivar y guiar a sus equipos en los tiempos de crisis).

Cabe decir que en otro estudio posterior, el CIPD (2009b) analiza a través de diez casos de estudio algunas de las prácticas de GT que las empresas han implementado en épocas de recesión. En dicho estudio se considera la alineación de recursos y prioridades y, la congelación tanto del reclutamiento como del salario, como respuestas habituales de las empresas del sector privado ante la crisis. Asimismo, se apunta a un estrecho control de gastos como una constante en dichas organizaciones.
Dicho estudio concluye con una serie de recomendaciones que se pueden resumir en las siguientes (CIPD, 2009b):

- Apostar por el desarrollo y formación de los líderes para saber actuar en época de crisis;

- Consolidar las habilidades de los jefes de línea en cuanto a la detección, desarrollo y evaluación del talento de sus subordinados (tales como performance management, saber dar una retroalimentación y llevar conversaciones difíciles, saber ofrecer coaching y motivar);

- Apoyar y mantener totalmente informados a los trabajadores de la situación real de la empresa (comunicar que está pasando y porqué, y mantener la atención sobre el clima de la organización);

- Pensar y actuar de forma holística vinculando el diseño organizativo, la gestión del talento, el performance management y el compromiso.

- Permanecer en contacto con la gente con talento que se haya acogido a sabáticos y/o excedencias con el fin de poder recuperarla en un futuro.

Así pues y, dado que la actual crisis económica tiene un alcance global, se considera interesante no sólo conocer la percepción del rol del departamento de RR.HH. en las empresas españolas, sino también la reacción de éstas ante la crisis en cuanto al enfoque de GT que utilizan. A continuación, se exponen los resultados obtenidos a través del panel de expertos sobre del concepto de talento y de la gestión del mismo en el contexto de las empresas españolas. 


\section{Conceptuación y ubicación de talento en las empresas españolas}

La revisión de la literatura sobre gestión del talento, si bien revela una falta de claridad y consenso sobre la definición y alcance del término talento, también apunta hacia una clara asociación entre habilidades y/o competencias y desempeño. También fue ese el caso de nuestros expertos. Tras analizar y contrastar sus definiciones de talento se pueden agrupar en dos de las concepciones identificadas en el barrido de la literatura:

- Talento entendido como un grupo de personas con un alto rendimiento y/o potencial. Pese a que sólo uno de los expertos consultados definió talento en estos términos, se considera interesante resaltar que su definición se ajusta perfectamente a lo encontrado en la literatura. Es más, trata al talento como un recurso estratégico, coincidiendo con algunos investigadores (por ejemplo: Seifert y Hadida, 2006; Holland et al., 2007) que utilizan la teoría de recursos y capacidades como marco teórico para discutir sobre el mismo.

"Talento son las personas que forman una empresa que aportan un valor diferencial para el cliente, el producto y el mercado, y que participa del elemento diferenciador frente a su competencia, aportando valor interno y externo". Experto $A$.

- Talento entendido como habilidades, capacidades y/o competencias de una persona. Sin lugar a dudas, esta ha sido la interpretación dominante en nuestro panel. La vinculación de dichas habilidades con resultados excelentes o extraordinarios, también ha sido una constante. A continuación, mostramos algunas de las definiciones:

"Habilidad practicada con interés y observable a través de resultados excelentes". Experto B.

"Capacidad de hacer las cosas obteniendo resultados extraordinarios". Experto $\mathrm{C}$.

"Independientemente del puesto desempeñado, es la adecuada conjunción de conocimientos, habilidades y actitudes que provocan un desempeño excelente y diferenciador, reportando al individuo la capacidad de detectar oportunidades no perceptibles para los demás, y construyendo donde otros consideran que el terreno es poco fértil". Experto $D$.

- Incluso algún experto menciona el hecho de que el talento pueda ser innato o adquirido:

"Es el conjunto de aptitudes o características, sobresalientes respecto de un grupo de personas, que permiten realizar una tarea de forma exitosa y que una persona puede o no llegar a desarrollar y hacerlo a un ritmo $u$ otro en función de las variables que se pueda encontrar en su desempeño. Puede ser heredado o adquirido". Experto $\mathrm{E}$.

- Pero, quizás lo más relevante sea mencionar que un experto ha definido el talento en forma de ecuación, introduciendo el compromiso como variable multiplicativa, tal y como hicieron Ulrich y Cubeiro.

"Capacitación x Compromiso x Ajuste estrategia compañía”. Experto F. 
Gestión del talento en la empresa española. Rol del departamento de RR.HH.

Gallardo-Gallardo, E.; González-Cruz, T.; Martínez-Fuentes, C. y Pardo-del-Val, M.

- Asimismo, la alineación de la persona con la organización es un tema clave, según ellos, al hablar de talento en una organización. Véanse, las siguientes definiciones:

"La capacidad para desempeñar una función en la empresa (operativa o directiva) y alcanzar objetivos con resultados que se ajustan o superan las expectativas de la compañía y de manera que se respeten la misión, valores, políticas y procedimientos de la empresa". Experto G.

"Empleado con conocimientos suficientes más las capacidades correspondientes de experiencia y valores multiplicado por el COMPROMISO". Experto $\mathrm{H}$.

Luego, los expertos consideran que no se puede equiparar talento al total de las personas de la organización, tal y como se puede deducir del siguiente enunciado emitido por uno de los expertos consultados: "En general existe muy poco talento en las compañías, no se trata de un tema de posición en el organigrama, depende de personas concretas".

Al preguntar a los expertos dónde consideran que se concentra el talento en las empresas españolas señalan aquellos puestos de mayor responsabilidad tanto directiva como técnica, los de mayor grado de experiencia y cualificación y, los más cercanos al cliente (véase, Tabla 1).

En una pregunta abierta posterior, dichos expertos apuntan a los departamentos técnicos y de I+D+i, equipos comerciales y directivos, y departamentos de supply chain y de producción, cómo aquellos en los que se concentra el talento actualmente. Asimismo, señalan a los departamentos de I+D+i, ventas y servicio al cliente, así como a los puestos de

Tabla 1

El talento se concentra en...

\begin{tabular}{lcccc}
\hline & \multicolumn{2}{c}{ Ronda 1 } & \multicolumn{2}{c}{ Ronda 2 } \\
\cline { 2 - 5 } & Mediana & Recorrido* & Mediana & Recorrido \\
\hline Los puestos de mayor responsabilidad directiva & 4 & 1,5 & 4,5 & 1 \\
Los puestos de mayor responsabilidad técnica & 4 & 0 & 4 & 0 \\
Los puestos de mayor grado de experiencia & 4 & 1,5 & 4 & 1 \\
Los puestos de mayor grado de cualificación & 4 & 1 & 4 & 0,5 \\
$\begin{array}{l}\text { Los puestos más cercanos a las actividades de } \\
\text { producción }\end{array}$ & 3 & 1 & 4 & 1,25 \\
Los puestos más cercanos al cliente & 4 & 1,5 & 3 & 0,25 \\
Los puestos más cercanos a las actividades de & 3 & 2 & 3 & 1 \\
I+D+i & & & & 1 \\
Los puestos más cercanos a las actividades de \\
aprovisionamiento
\end{tabular}

* Las columnas de Recorrido, de ahora en adelante, hacen referencia al recorrido intercuartílico entre los expertos. Fuente: Elaboración propia. 
alta dirección y consejos de administración, como los puestos dónde debería producirse el acopio de talento en los próximos años.

\section{Rol y prácticas del departamento de RR.HH. en las empresas españolas}

En la Tabla 2 se recogen las opiniones de los expertos sobre la relación entre los departamentos de RR.HH. y la estrategia de la empresa. Si bien los departamentos de RR.HH. comprenden la estrategia de la empresa y los factores clave de éxito, no juegan un papel clave en el diseño de la estrategia de la misma. Por tanto, no es de extrañar que los expertos afirmen no observar una clara vinculación entre las actividades de estos departamentos y la consecución de la ventaja competitiva de la empresa.

Asimismo, según nuestros expertos, los departamentos de RR.HH. de las empresas españolas no conocen ni comprenden perfectamente los roles críticos y las competencias requeridas. Hecho que no sólo refuerza la percepción de una desconexión entre las actividades de estos departamentos y la consecución de ventajas competitivas, sino que apunta hacia unas políticas y prácticas de RR.HH. de corte clásico (sin otorgar a la DRR.HH. un carácter estratégico).

Es interesante resaltar el hecho de que las acciones innovadoras que emprenden los departamentos de RR.HH son consideradas "copias" de las mejores prácticas que están de moda (véase Tabla 3). Además, las acciones que emprenden tienen un marcado carácter exclusivista (obtener resultados extraordinarios o un alto desempeño es clave). No obstante, y en línea con lo mencionado anteriormente, no existe una visión estratégica de puestos y competencias clave para la empresa. La inexistencia de programas de incorporación de personal con

Tabla 2

Los departamentos de RR.HH. y la estrategia de la empresa

\begin{tabular}{lcccc}
\hline & \multicolumn{2}{c}{ Ronda 1 } & \multicolumn{2}{c}{ Ronda 2 } \\
\cline { 2 - 5 } & Mediana & Recorrido & Mediana & Recorrido \\
\hline $\begin{array}{l}\text { No se observa un conocimiento claro de la rela- } \\
\text { ción causal que conecta las actividades de }\end{array}$ & 4 & 1,5 & 4 & 1 \\
$\begin{array}{l}\text { RR.HH. con la consecución de la ventaja compe- } \\
\text { titiva de la empresa }\end{array}$ & & & \\
$\begin{array}{l}\text { Los departamentos de RR.HH. comprenden la } \\
\text { estrategia de la empresa y los factores clave de }\end{array}$ & 4 & 2 & 3 & 0,25 \\
éxito \\
$\begin{array}{l}\text { Los departamentos de RR.HH. conocen y com- } \\
\text { prenden los roles críticos y las competencias re- } \\
\text { queridas }\end{array}$ \\
$\begin{array}{l}\text { RR.HH. es un elemento clave en el diseño de la } \\
\text { estrategia general de la empresa }\end{array}$ & 2 & & & \\
\hline
\end{tabular}

Fuente: Elaboración propia. 
Gestión del talento en la empresa española. Rol del departamento de RR.HH.

Gallardo-Gallardo, E.; González-Cruz, T.; Martínez-Fuentes, C. y Pardo-del-Val, M.

Tabla 3

Las prácticas del departamento de RR.HH.

\begin{tabular}{|c|c|c|c|c|}
\hline & \multicolumn{2}{|c|}{ Ronda 1} & \multicolumn{2}{|c|}{ Ronda 2} \\
\hline & Mediana & Recorrido & Mediana & Recorrido \\
\hline $\begin{array}{l}\text { Las acciones innovadoras en RR.HH. son copia de las } \\
\text { "mejores prácticas" que están de moda. }\end{array}$ & 4 & 0 & 4 & 0 \\
\hline $\begin{array}{l}\text { Los indicadores financieros del departamento de } \\
\text { RR.HH. están centrados en el control de costes }\end{array}$ & 4 & 0 & 4 & 0,25 \\
\hline $\begin{array}{l}\text { Los incentivos y recompensas se dan sólo a quienes } \\
\text { alcanzan resultados extraordinarios }\end{array}$ & 4 & 1 & 4 & 1 \\
\hline $\begin{array}{l}\text { Aportar nuevas ideas y un alto desempeño es el crite- } \\
\text { rio para promocionar a una persona }\end{array}$ & 4 & 1,5 & 3,5 & 1 \\
\hline $\begin{array}{l}\text { Sólo hay que invertir en el desarrollo de las personas } \\
\text { que ocupan puestos críticos o en aquellas que tienen } \\
\text { potencial para hacerlo }\end{array}$ & 3 & 1,5 & 3,5 & 1 \\
\hline $\begin{array}{l}\text { La contratación de personas con capacidades críticas } \\
\text { es una prioridad }\end{array}$ & 3 & 0 & 3 & 0,25 \\
\hline $\begin{array}{l}\text { Se considera que el desempeño de todos los trabaja- } \\
\text { dores debe mejorar año a año }\end{array}$ & 3 & 1 & 3 & 0,25 \\
\hline $\begin{array}{l}\text { El nivel de competencia y motivación de la plantilla es } \\
\text { responsabilidad de RR.HH. }\end{array}$ & 3 & 1 & 3 & 0,25 \\
\hline $\begin{array}{l}\text { Existen programas de reincorporación de personal } \\
\text { con competencias importantes para la empresa }\end{array}$ & 2 & 2 & 2 & 1,25 \\
\hline $\begin{array}{l}\text { Existen programas de desarrollo de personal enfoca- } \\
\text { dos especialmente a puestos clave para la empresa }\end{array}$ & 2 & 1,25 & 2 & 0,5 \\
\hline
\end{tabular}

competencias importantes y de programas de desarrollo de las personas que ocupan puestos clave lo demuestran. Priman resultados obtenidos independientemente de dónde se obtengan.

El panel de expertos afirma que la captación de nuevo personal con talento se basa, fundamentalmente, en la relación entre competencias y, tanto la ventaja competitiva como la estrategia de la empresa (véase Tabla 4). Siendo la disponibilidad en el mercado de esas personas con talento, el tercer criterio según ellos. Es interesante señalar que los temas relacionados con el salario aparecen en el último lugar.

\section{La gestión de personal en tiempos de crisis en las empresas españolas. Tendencias de futuro}

Dado que esta investigación se enmarca dentro de la mayor crisis económica de nuestro tiempo y, siendo conscientes del impacto que toda situación econó- 
mica delicada tiene sobre el tejido empresarial, se quiso conocer la opinión del panel acerca de cómo esta situación podía afectar a la forma en que las empresas españolas gestionan a su personal.

En una crisis lo primero que destaca, a nivel de empresas, es el estanca- miento en el reclutamiento de personal y el aumento de despidos, por lo que se preguntó a los expertos su percepción sobre estos temas haciendo referencia a la etapa anterior a la crisis (2000-2007) y a la actual etapa de crisis. El Gráfico 1 recoge dicha información.

Tabla 4

Las empresas valoran el talento de su personal o de los candidatos a serlo en función...

\begin{tabular}{lccccc}
\hline & \multicolumn{2}{c}{ Ronda 1 } & \multicolumn{2}{c}{ Ronda 2 } \\
\cline { 2 - 5 } & Mediana & Recorrido & Mediana & Recorrido \\
\hline $\begin{array}{l}\text { De la relación entre esas competencias y la ventaja } \\
\text { competitiva de la empresa }\end{array}$ & 5 & 2 & 5 & 1,25 \\
$\begin{array}{l}\text { De la importancia que tienen esas competencias para } \\
\text { la estrategia de la empresa }\end{array}$ & 4 & 1,5 & 4 & 1,25 \\
$\begin{array}{l}\text { De la disponibilidad en el mercado de personas con } \\
\text { esa cualificación/experiencia }\end{array}$ & 4 & 2 & 4 & 0,25 \\
$\begin{array}{l}\text { Del puesto jerárquico a ocupar } \\
\text { Del salario que están ofreciendo en otras empresas }\end{array}$ & 3 & 0,5 & 4 & 0,25 \\
De los salarios pagados por los competidores directos & 3 & 0 & 3 & 0,25 \\
\hline
\end{tabular}

Fuente: Elaboración propia.

\section{Gráfico 1}

Reclutamiento y selección en tiempos de crisis

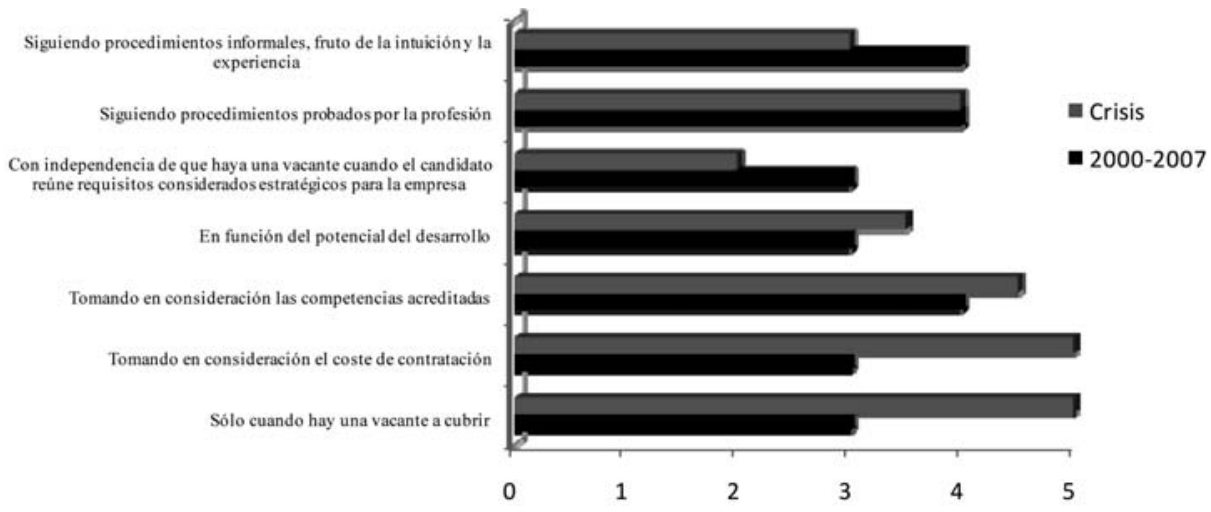

* Para realizar este gráfico se ha tenido presente la puntuación media obtenida en la segunda ronda de análisis. Cabe decir que salvo en el ítem "En función del potencial del desarrollo" las diferencias obtenidas son aceptables (hich $\leq 1$ ). Fuente: Elaboración propia. 
Gestión del talento en la empresa española. Rol del departamento de RR.HH.

Gallardo-Gallardo, E.; González-Cruz, T.; Martínez-Fuentes, C. y Pardo-del-Val, M.

Se puede observar cómo en época de crisis se contrata sólo cuando hay una vacante a cubrir y tomando en consideración el coste de contratación y las competencias acreditadas, reafirmando de este modo, la idea de que el departamento de $\mathrm{RR}$.HH. es un centro de coste y, para nada, un partner estratégico como propone Lawler (2008). Nótese cómo el ítem menos valorado y, por tanto, que indica que no se tiene en consideración al reclutar y seleccionar candidatos, es el hecho de que el candidato muestre requisitos considerados estratégicos para la empresa. Tampoco en el período previo a la crisis se observaban inquietudes a este respecto, lo que nos ratifica la idea de que el departamento de RR.HH. en la empresa española sigue teniendo un marcado carácter administrativo.
No obstante, cabe destacar la falta de acuerdo entre los expertos respecto a cómo afecta el potencial de desarrollo del individuo a las decisiones de reclutamiento y selección (el recorrido intercuartílico de las medianas es de 2).

Siguiendo en la misma línea, el Gráfico 2 presenta las respuestas de los expertos sobre los criterios de las empresas españolas a la hora de llevar a cabo despidos. Que el puesto no tenga la carga de trabajo necesaria para justificarlo y el coste del despido son los dos factores predominantes. Sin embargo, destaca la falta de acuerdo entre los expertos (recorrido de 2,25) en cuanto al rendimiento del individuo como base para el despido en época de crisis.

También se quiso conocer la opinión ${ }^{3}$ de los expertos sobre cómo podía

Gráfico 2

Razones para prescindir de personal

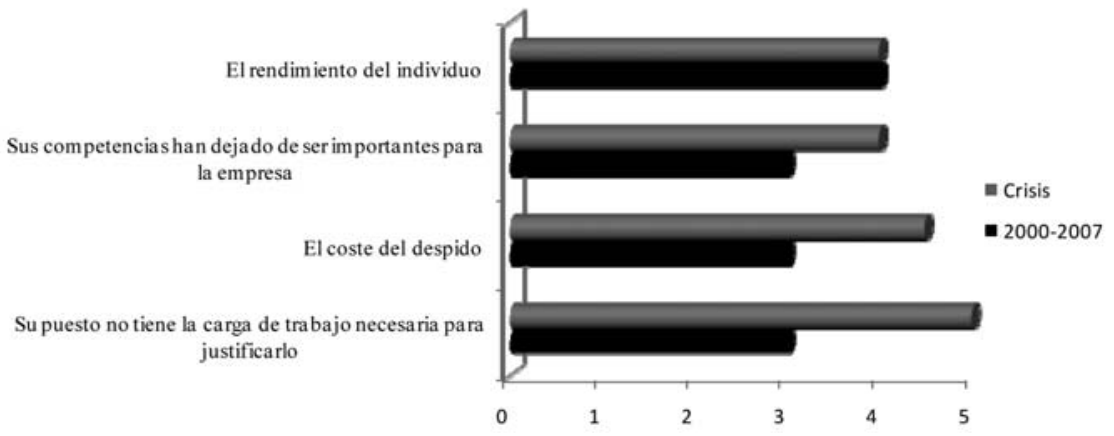

* Para realizar este gráfico se ha tenido presente la puntuación media obtenida en la segunda ronda de análisis. Cabe decir las diferencias obtenidas son aceptables (hich $\leq 1$ ), salvo en el ítem del rendimiento del individuo. Fuente: Elaboración propia.

3 En este caso, la escala de medida de cada uno de los ítems planteados fue una gradación de -3 a +3 ; indicando -3 un cambio negativo o reducción en ese rol y, +3 un cambio positivo o progresión. 
influir la actual situación económica en los roles del departamento de RR.HH. La Tabla 5 recoge sus opiniones. Si bien a todos los roles se les augura un cambio en positivo, tres son los que se considera que el departamento de RR.HH. reforzará en mayor medida: apoyar y aconsejar a los empleados; apoyar a los directivos de línea en la dirección de los RR.HH. y, apoyar y aconsejar al Consejo de Administración en temas concretos. Sin embargo, los expertos siguen sin apostar por un gran cambio (véase, mayor importancia en el rol) en cuanto al papel del departamento de RR.HH. en la fase de formula- ción y de implementación de la estrategia de la empresa.

Por último, se quiso averiguar su opinión sobre el posible cambio de enfoque de las empresas españolas respecto a la dirección de RR.HH. para afrontar la actual crisis ${ }^{4}$ (véase Tabla 6).

Nuevamente se observa un pronóstico de evolución positiva en todos los ítems contemplados. No obstante, destaca el enfoque hacia la eficiencia y el control de costes seguido de una reducción o ajuste de las condiciones laborales para todo el personal y un ajuste de 'forma poco traumática' de los excedentes de

Tabla 5

Influencia de la crisis económica en los roles del departamento de RR.HH.

\begin{tabular}{lcccc}
\hline & \multicolumn{2}{c}{ Ronda 1 } & \multicolumn{2}{c}{ Ronda 2 } \\
\cline { 2 - 5 } & Mediana & Recorrido & Mediana & Recorrido \\
\hline Apoyar y aconsejar a los empleados & 3 & 1 & 3 & 1 \\
$\begin{array}{l}\text { Apoyo a los directivos de línea en la dirección de } \\
\text { los RR.HH. }\end{array}$ & 2 & 2 & 2 & 1,25 \\
$\begin{array}{l}\text { Apoyar y aconsejar al Consejo de Administración } \\
\text { en temas concretos (remuneración y contratación } \\
\text { de nuevos directivos; planes estratégicos de ad- } \\
\text { quisición de competencias, etc.) }\end{array}$ & 2 & 2,25 & 2 & 1,25 \\
$\begin{array}{l}\text { Participante de primer nivel en el proceso de for- } \\
\text { mulación de la estrategia }\end{array}$ & 1,5 & 1,25 & 1,5 & 1 \\
$\begin{array}{l}\text { Proveedor de herramientas y recursos de gestión } \\
\text { de RR.HH. }\end{array}$ & 1 & 2,5 & 1,5 & 2,25 \\
$\begin{array}{l}\text { Agente clave en el proceso de implementación } \\
\text { (ejecución) de la estrategia }\end{array}$ & 1 & 1,5 & 1 & 1,25 \\
\hline
\end{tabular}

Fuente: Elaboración propia.

4 De nuevo, la forma de valoración fue una gradación de las respuestas desde -3 (cambio negativo o reducción) a +3 (cambio positivo o progresión). 
Gestión del talento en la empresa española. Rol del departamento de RR.HH.

Gallardo-Gallardo, E.; González-Cruz, T.; Martínez-Fuentes, C. y Pardo-del-Val, M.

Tabla 6

La dirección de RR.HH. ante la crisis

\begin{tabular}{|c|c|c|c|c|}
\hline & \multicolumn{2}{|c|}{ Ronda 1} & \multicolumn{2}{|c|}{ Ronda 2} \\
\hline & Mediana & Recorrido & Mediana & Recorrido \\
\hline Enfoque hacia la eficiencia y el control de coste & 2,5 & 1,25 & 2,5 & 1 \\
\hline $\begin{array}{l}\text { Reducción/ajuste de las condiciones laborales } \\
\text { para todo el personal }\end{array}$ & 2 & 0,5 & 2 & 0,5 \\
\hline $\begin{array}{l}\text { Ajustar de forma 'poco traumática' los exceden- } \\
\text { tes de plantilla }\end{array}$ & 2 & 1,75 & 2 & 1,5 \\
\hline $\begin{array}{l}\text { Interés por contratar y retener al personal con } \\
\text { competencias clave para la empresa }\end{array}$ & 2 & 3,5 & 2 & 2,75 \\
\hline $\begin{array}{l}\text { Buscar soluciones imaginativas para no despren- } \\
\text { derse de las personas con talento reclutadas en } \\
\text { los años precedentes }\end{array}$ & 1,5 & 1,25 & 1,5 & 1,25 \\
\hline $\begin{array}{l}\text { Reducción/ajuste de las condiciones laborales } \\
\text { para el personal no estratégico }\end{array}$ & 1,5 & 2 & 1,5 & 2 \\
\hline $\begin{array}{l}\text { Mayor exigencia de un papel clave del departa- } \\
\text { mento de RR.HH. en la formulación de las estra- } \\
\text { tegias de futuro }\end{array}$ & 1,5 & 3,5 & 1,5 & 2 \\
\hline $\begin{array}{l}\text { Desarrollo de la imagen de empleador para atraer } \\
\text { talento excedente de otras empresas }\end{array}$ & 0,5 & 2,75 & 1 & 2,75 \\
\hline
\end{tabular}

plantilla. En otras palabras, la dirección de RR.HH. tiene que conseguir minimizar el coste de personal en estos años de crisis, eso sí, deberá plantearse soluciones creativas para retener al personal con talento y ajustar las condiciones laborales para el personal no estratégico (si bien el grado de desacuerdo en esto último es bastante elevado).

Llama la atención la controversia que genera entre los expertos consultados el interés por contratar y retener al personal con competencias clave para la empresa, lo que nos demuestra que sigue sin estar claro el rol estratégico que debería ejercer la dirección de RR.HH.

\section{Conclusiones}

A continuación expondremos las conclusiones que pueden derivarse de las reflexiones planteadas a lo largo del artículo. La primera conclusión subraya la importancia de la conceptuación de "talento" para avanzar en el campo de la GT ya que la confusión imperante sobre qué se entiende por talento en las empresas está obstaculizando el establecimiento de teorías y prácticas de GT. ¿Cómo se puede hablar de gestionar talento y de prácticas de GT si no se ha llegado a un consenso sobre qué es talento?

La segunda conclusión deriva del análisis de la literatura realizado e indica 
la existencia de tres grandes interpretaciones del término talento en el mundo empresarial: talento entendido como el total de personas de una organización; talento entendido como un conjunto de personas de una organización con un alto rendimiento y/o potencial; y, por último, talento entendido como aquellas habilidades, capacidades y/o competencias de una persona que le permiten conseguir resultados extraordinarios. Sin duda, esta es una aportación importante del artículo ya que se han identificado las tres grandes acepciones del término talento existentes en la literatura hasta el momento. Siendo éstas las que explican las diferentes estrategias de GT existentes $y$, también, las que pueden pautar las futuras líneas de investigación en este campo.

La tercera conclusión se deriva de la consulta a expertos realizada y plantea que en las empresas españolas se entiende mayoritariamente el talento como aquellas habilidades, conocimientos $y$ actitudes de una persona que le permiten obtener unos resultados excelentes. Al parecer no se le concede una importancia primaria a la cuestión del carácter innato del talento y resalta el compromiso como componente actitudinal clave. Una reflexión adyacente que se infiere de esta discusión es que: si talento no es sinónimo de personas de la organización, la GT se antoja diferente a la dirección de RR.HH. Sin duda, aquí se vislumbra una línea de investigación futura.

La cuarta conclusión ratifica el papel administrativo del departamento de $\mathrm{RR}$.HH. en las empresas españolas y su caracterización como un centro de costes discrecionales, donde sus indicadores están centrados en la eficiencia y no tanto en la eficacia de las acciones realizadas. Destaca la falta de conexión con la estrategia y el desconocimiento acerca del impacto de las prácticas de RR.HH. sobre los resultados de la organización. En consecuencia, el papel del departamento de RR.HH. en el proceso de formulación de la estrategia sigue siendo secundario y su comprensión de los factores clave de éxito limitada -así como de su traslación en términos de competencias de los individuos- para la consecución de ventajas competitivas.

Por último, la quinta conclusión hace referencia a las prácticas de RR.HH. utilizadas en las empresas españolas. En línea con lo expuesto anteriormente, se infiere la clara ausencia de un enfoque propio 'ajustado a la estrategia'. Es decir, se siguen las modas que en cada momento son etiquetadas como ' $\mathrm{m}$ ejores prácticas' y destaca la escasez de prácticas orientadas a la captación, desarrollo o recuperación de talento. Asimismo, en la medida en la que el departamento de RR.HH. es visto como un centro de costes discrecionales, el criterio fundamental de contratación y despido en las empresas españolas es el coste, relegando a un segundo lugar aquellos procesos que toman en consideración si el candidato posee competencias estratégicas para la empresa.

Del trabajo presentado se extraen una serie de recomendaciones para gestionar el talento en las empresas españolas, si bien, se han de interpretar con la prudencia correspondiente al ser éste de carácter exploratorio. Se debe entender talento como algo propio de las personas. Concretamente, ciertas habilidades o 
Gestión del talento en la empresa española. Rol del departamento de RR.HH.

Gallardo-Gallardo, E.; González-Cruz, T.; Martínez-Fuentes, C. y Pardo-del-Val, M.

competencias que permiten a las personas comprometidas con su trabajo obtener unos resultados extraordinarios. Así, a la hora de gestionarlo, los directivos deben saber que el talento: (a) es identificable por un excelente desempeño y/o potencial; (b) no se trata exclusivamente de habilidades innatas sino que, en parte, se puede desarrollar; (c) está muy vinculado a las actitudes, en concreto al compromiso de la persona con la tarea realizada.

La organización puede gestionar el talento de sus empleados, a través de los enfoques basados en las competencias, mediante programas personalizados de formación y desarrollo de habilidades (por ejemplo, coaching y mentoring), retroalimentaciones de desempeño periódicas y completas (feedback $360^{\circ}$, sesiones de evaluación del talento) y, prácticas que favorezcan y refuercen el compromiso individual.

Asimismo, el departamento de $\mathrm{RR}$.HH. debería asumir un rol de carácter estratégico. Esto pasa por: a) un cambio de mentalidad en los directivos de las empresas españolas; b) una mejor comprensión de las estrategia y de los factores clave de éxito por parte de los directivos de RR.HH.; c) el desarrollo de sistemas de información que permitan conocer y vincular las prácticas de RR.HH. con el desempeño de la empresa. Aunque este cambio de rol resulta aún más importante en la actual época de crisis, parece difícil que produzca, ya que las empresas se centran especialmente en la eficiencia económica a corto plazo.

\section{Referencias bibliográficas}

Blass, Eddie (2009). Talent Management: Cases and Commentary, Hampshire, Palgrave Macmillan.

Collings, David G. y Mellahi, Kamel (2009). Strategic talent management: A review and research agenda, Human Resource Management Review, Vol. 19, No 4, pp. 304-313.

Chartered Institute of Personnel and Development (CIPD) (2007). Talent management. Research insight, http://www. cipd.co.uk/NR/rdonlyres/B513502C8F42-419C-818C-D3C12D87E0D3/ 0/talentmanage. pdf, consultado el 15 de Junio de 2010.

Chartered Institute of Personnel and Development (CIPD) (2009a). The war on talent? Talent managementunder threat in uncertain times. Part I. Survey Report. February 2009, http://www.cipd. co.uk/NR/rdonlyres/DFC760F8-F37 C-4548-9470-154073FE4EC/0/warontalent.pdf, consultado el 15 de Junio de 2010.

Chartered Institute of Personnel and Development (CIPD) (2009b). Fighting back through talent innovation: Talent management under threat in uncertain times. Part II. Report. July 2009, http:// www.cipd.co.uk/NR/rdonlyres/D5B78 09A-FBF0-489D-BB73-26C0C3C7B 194/0/Fighting_back_through_talent _innovation.pdf, consultado el 15 de Junio de 2010.

Cheese, Peter, Thomas, Robert J. y Craig, Elisabeth (2008). The talent powered organization: Strategies for globalization, talent management and high performance. Londres, Kogan Page.

Cubeiro, Juan Carlos (2008). Presente y futuro del desarrollo de los talentos en España, en Blanco, J. (coord.), Desa- 
rrollo del talento, Madrid, AEDIPE Centro and Pearson Educación, S.A.

Dalkey, Norman C. y Helmer, Olaf (1963). An experimental application of the Delphi Method to the use of experts, Management Science, Vol. 9, Abril, pp. 458-467.

Dalkey, Norman C., Brown, Bernice y Cochran, S.W. (1970). The Delphi Method, III: Use of self-rating to improve group estimates, Technological Forecasting and Social Change, No 1, pp. 283-291.

Effron, Marc y Ort, Miriam (2010): One page talent management: Eliminating complexity, adding value, Boston, Harvard Business Press.

Farley, Cheryl (2005). HR's role in talent management and driving business results, Employment Relations Today, Vol. 32, No 1, pp. 55-61.

Goffee, Rob, y Jones, Gareth (2007). Leading clever people. Harvard Business Review, Vol. 85, No 3, pp. 72-79.

González-Cruz, Tomás, Martínez-Fuentes, Clara y Pardo-del-Val, Manuela (2009). La gestión del talento en la empresa industrial española, Economía Industrial, No 374, pp. 21-35.

Gubman, Edward L. (1998). The talent solution: Aligning strategy and people to achieve extraordinary results, Nueva York, McGraw-Hill.

Iles, Paul, Chuai, Xin y Preece, David (2010). Talent Management and HRM in Multinational companies in Beijing: Definitions, differences and drivers, Journal of World Business, Vol. 45, pp. 179-189.

Lawler III, Edward. E. (2008). Talent: Making people your competitive advantage, San Francisco, Jossey-Bass.

Lewis, Robert E. y Heckman, Robert J. (2006): Talent Management: A critical review. Human Resource Management Review, vol. $16, n^{\circ} 2$, págs. 139-154.
Luna, Roberto (2010). Top-Talento: Estudio de la gestión del talento en la empresa española. http://www.robertoluna.es/ descargas/conclusiones_estudio_ gesta.pdf?phpMyAdmin=vWyRwhw nmTkYISHPGAPwUbFJ4p8, consultado el 11 de febrero de 2011.

Michaels, Ed; Handfield-Jones; Helen y Axelrod, Beth (2001): The war for talent, Boston, Harvard Business School Press.

O'Reilly, Charles A. y Pfeffer, Jeffrey (2000). Cisco Systems: Acquiring and retaining talent in hypercompetitive markets, Human Resource Planning, Vol. 23, No 3, pp. 38-52.

Pfeffer, Jeffrey (2001). Fighting the war for talent is hazardous to your organization's health, Organizational Dynamics, Vol. 29, No 4, pp. 248-259.

Seifert, Matthias y Hadida, Allegre L. (2006). Facilitating talent selection decisions in the music industry, Management Decision, Vol. 44, No 6, pp. 790-808.

Silzer, Rob y Dowell, Ben E. (eds.) (2010). Strategy-Driven Talent Management: A leadership imperative. John Wiley \& Sons, San Francisco.

Stahl, Günter K., Björkman, Ingmar, Farndale, Elaine, Morris, Shad S., Paauwe, Jaap, Stiles, Philip, Trevor, Jonathan y Wright, Patrick M. (2007). Global talent management: How leading multinational build and sustain their talent pipeline, INSEAD Faculty and Research Working Papers, 2007/24/ OB.

Tansley, Carole, Turner, Paul, Foster, Carley, Harris, Lynette, Sempik, Anne, Stewart, Jim y Williams, Hazel (2007). Talent: strategy, management, measurement, Londres Chartered Institute of Personnel and Development.

The Economist (2006). The battle for brainpower, 5 de Octubre. 
Gestión del talento en la empresa española. Rol del departamento de RR.HH.

Gallardo-Gallardo, E.; González-Cruz, T.; Martínez-Fuentes, C. y Pardo-del-Val, M.

Thorne, Kaye y Pellant, Andy (2007). The essential guide to managing talent. How top companies recruit, train \& retain the best employees, London, Kogan Page.

Ulrich, Dave (2007). The talent trifecta, Workforce Management, Vol. 86, No 15 (Octubre), http://www.workforce.com/ section/01/feature/25/12/27/in- dex printer.htmlconsultado el 12 de Agosto de 2010.

Ulrich, Dave y Brockbank, Wayne (2005). The HR Value Proposition, Boston, Harvard Business School Press.

Ulrich, Dave y Smallwood, Norm (2005). HR's new ROI: Return on Intangibles, Human Resource Management, Vol. 44, No 2, pp. 137-142. 